\begin{tabular}{|c|c|c|c|}
\hline i.e.t.c.c. & \multicolumn{2}{|c|}{$\begin{array}{l}\text { Materiales y procedimientos no tradicionales de construcción } \\
\text { DOCUMENTO DE IDONEIDAD TÉCNICA }\end{array}$} & C.D.U.: 69.022 .3 \\
\hline \multirow{2}{*}{$\begin{array}{l}\text { Instituto } \\
\text { Eduardo tormoja } \\
\text { Costillares - Chamartín } \\
\text { MADRID-16-ESPAÑA }\end{array}$} & \multirow{2}{*}{\multicolumn{2}{|c|}{$\begin{array}{l}\text { Fabricante: } \\
\text { Sociedad Anónima de Materiales y Obras SAMO } \\
\text { Dom. Social: Colón, } 68 \\
\text { Fábrica: MISLATA - VALENCIA }\end{array}$}} & D. I. T. N. 11 \\
\hline & & & $\begin{array}{l}\text { Elementos para } \\
\text { muros } \\
\text { Éléments pour murs } \\
\text { Elements for walls }\end{array}$ \\
\hline
\end{tabular}

\section{INFORIME TECNICO}

\section{MATERIAL}

\subsection{Definición}

EI YTONG-0,65, fabricado por la casa SAMO, de Valencia, es un hormigón del grupo de los hormigones ligeros a la cal. Tiene estructura porosa, constituida por células esferoidales estancas cuyas dimensiones dependen de la densidad; las delgadas paredes de estas células tienen como principal componente el hidrosilicato de cal formado por tratamiento al vapor.

\section{FABRICACION}

\subsection{Materias primas}

Las materias primas utilizadas son: el "rodeno", roca arenisca con gran proporción de sílice, y la cal grasa; por otra parte, se utiliza también polvo de aluminio.

\subsection{Maquinaria empleada en la fabricación}

Esencialmente la maquinaria que interviene en el proceso de fabricación es la siguiente:

- Sección de molido: machacadora, molinos de martillos y molinos de bolas con los consiguientes elevadores y transportadores helicoidales para distribución de materiales en silos o tolvas. Para el transporte de la harina a la zona de mezcla se utiliza un procedimiento neumático.

- Sección de mezclado: consta de tolvas, básculas y una mezcladora.

- Sección de curado y corte: máquina cortadora, vagonetas y moldes.

- Sección de curado: autoclave y generador de vapor.

La maquinaria es totalmente nacional. Se exceptúa la máquina cortadora, que es de procedencia sueca.

\subsection{Proceso}

La roca silícea y la cal grasa se molturan por separado y posteriormente se dosifican estos pro- ductos, mezclándose y homogeneizándose. A continuación se les añade agua y polvo de aluminio. La masa flúida que así se obtiene se vierte en moldes metálicos cuyas dimensiones son: $2,40 \times$ $\times 1,70 \times 0,49 \mathrm{~m}$ en los que las burbujas de gas desprendidas por la acción del polvo de aluminio, hacen aumentar su volumen hasta producir un ligero desbordamiento. Al cabo de cierto tiempo de reposo, el material adquiere una consistencia tal que permite cortarlo en bloques de tamaño adecuado. A continuación, se introducen en la autoclave, donde se someten a la acción del vapor de agua a una presión de $10 \mathrm{~kg} / \mathrm{cm}^{2}$ que les confiere propiedades finales de dureza, resistencia y estabilidad.

Finalmente, los bloques se sacan de los moldes y se almacenan a cubierto.

\subsection{Controles}

a) De las materias primas

Independientemente del laboratorio general, existe un laboratorio complementario cuya misión exclusiva es el análisis sistemático de las materias primas, especialmente de la riqueza de cal, ya que el rodeno tiene una composición de sílice casi constante. Las muestras de las materias primas, cal y rodeno se someten, en el momento de su descarga, a análisis químico para control de su pureza. Igualmente se hace con el aluminio, determinando velocidades de reacción.

\section{b) Fabricación}

El control de fabricación se efectúa durante el proceso, determinándose: finura de los productos molidos, temperaturas y dosificación en el amasado, temperatura de curado al aire y temperatura y presiones en el curado al vapor.

c) Producto acabado

El producto acabado se somete normalmente a los ensayos de densidad, resistencia a compresión y absorción de agua; y periódicamente se determina la retracción por desecado.

Para estos ensayos se obtienen dos bloques de muestra, uno del centro y otro del borde, en tres moldes de cada autoclave. De cada bloque se cortan unas probetas cúbicas de $10 \mathrm{~cm}$ de lado, $\mathrm{y}$ otras prismáticas, secándose artificialmente hasta 
un 5 o un 10 por 100 de humedad relativa/volumen. Las probetas cúbicas se ensayan a compresión hasta la rotura, y las probetas prismáticas se terminan de secar a $100^{\circ} \mathrm{C}$ hasta peso constante, para determinar la densidad en dicho estado. La media de estos ensayos se refleja en el parte correspondiente.

Para determinar la retracción se utiliza un armario con circulación constante de aire y grado de humedad determinado, donde se colocan las probetas saturadas de agua, midiéndose la longitud de las mismas antes de meterlas en el armario, y después de haber alcanzado el equilibrio con la humedad del aire en circulación.

\section{ELEMENTOS}

\subsection{Bloques}

Los bloques tienen dimensiones nominales de $49 \times 24 \mathrm{~cm}$ y espesores de $15,18,21$ y $24 \mathrm{~cm}$ Las dimensiones técnicas de fabricación son $490 \times 238 \mathrm{~mm}$, y los espesores, de 148, 178, 208 y 238 milímetros.

\section{PUESTA EN OBRA DEL MATERIAI}

4.1. Recomendaciones y características generales de ejecución y acabado de la albañilería

En general, la puesta en obra del YTONG-0,65 se rige por las normas tradicionales de la buena albañilería.

Los aparejos en las fábricas con YTONG-0,65 son los normales en la construcción, obteniéndose los diferentes espesores con los distintos tipos que se fabrican. Las juntas deben ser de $1 \mathrm{~cm}$, teniendo en cuenta sus dimensiones.

Para la puesta en obra del YTONG-0,65 es conveniente mojarlo en el momento de su utilización. En tiempo normal es suficiente que el agua penetre $5 \mathrm{~mm}$ a partir de la superficie de juntas, pero en tiempo seco se deberá humedecer hasta 8 ó 10 milímetros de profundidad para evitar el riesgo de deshidratación del mortero.

Los bloques de YTONG-0,65 se pueden cortar con los corta-bloques y corta-placas especiales, como igualmente aserrar con un serrucho de púas gruesas o trabajar con el hacha o formón.
Con aserrados sucesivos se pueden preparar las mochetas necesarias para la colocación de los marcos de ventanas o puertas.

Las especiales cualidades del YTONG-0,65 permiten la ejecución, por procedimientos muy simples, de las rozas para empotrar la instalación eléctrica, así como de los huecos para colocar los cajetines de empalmes o interruptores.

Igualmente se puede atornillar directamente sin necesidad de tacos.

\subsection{Morteros}

Para la ejecución de fábricas de bloques YTONG, se pueden utilizar los morteros que habitualmente se emplean en albañilería, recomendándose que, dada la marcada rugosidad de las superficies de este material, dichos morteros sean de consistencia plástica.

En la ejecución de la tabiquería se pueden utilizar las pastas de yeso como elemento de unión.

\subsection{Revestimientos}

Para la ejecución de los revestimientos pueden utilizarse los mismos morteros que para la ejecución de las fábricas de bloques.

Es conveniente dejar secar la fábrica antes de su ejecución, así como frotar las superficies a revestir con un cepillo de púas metálicas $u$ otro similar, con objeto de quitar las pequeñas porciones de material que hayan quedado sueltas después del cortado en fábrica. Se debe mojar la superficie a revestir, incluso la noche anterior si el tiempo es seco. Esta superficie debe estar húmeda en una profundidad de unos 5 milímetros.

\section{CARACTERISTICAS Y ENSAYOS}

Este material ha sido sometido a ensayo por el Instituto Eduardo Torroja de la Construcción y del Cemento, y las características que figuran en el Cuadro adjunto son el resultado de los mismos. En este Cuadro se recogen también los valores exigidos por las «reglas para apreciación técnica de bloques para muros, de hormigón ligero, tratados al autoclave».

\begin{tabular}{|l|l|c|c|}
\hline \multicolumn{1}{|c|}{ C A R A C T E R I S T I C A S } & $\begin{array}{c}\text { Resultado } \\
\text { de los } \\
\text { ensayos }\end{array}$ & Reglas \\
\hline & Precisión dimensional & $\pm 2 \mathrm{~mm}$ & $\pm 3 \mathrm{~mm}$ \\
\hline \multirow{2}{*}{$\begin{array}{l}\text { Resistencia } \\
\text { a compresión }\end{array}$} & Resistencia en $\mathrm{kg} / \mathrm{cm}^{2}$ & 76,9 & 50 \\
\hline & Dispersión de los resultados (coeficiente de variación en \%) & 13,9 & 15 \\
\hline \multirow{2}{*}{\begin{tabular}{l} 
Densidad \\
\cline { 2 - 5 }
\end{tabular}} & Diferencia entre densidad nominal y en estado seco & 0,017 & $\pm 0,05$ \\
\hline & Dispersión de los resultados (coeficiente de variación en \%) & 2,6 & 3 \\
\hline \multicolumn{2}{|c|}{ Variación dimensional higrométrica en mm/m } & 0,43 & 0,65 \\
\hline
\end{tabular}




\section{REFERENCIAS DE UTILIZACION}

En la actualidad han sido fabricados más de $250.000 \mathrm{~m}^{3}$ de YTONG en España, habiéndose utilizado principalmente en viviendas y en instalaciones industriales. Se ha utilizado tanto en muros resistentes como en muros de cerramientos sin carga y en revestimientos térmicos.

Entre las principales obras ejecutadas figuran:

1. 1.500 viviendas en Vistabella (Murcia), para la Obra Sindical del Hogar (Empresa Bernal Pareja).

En estas viviendas se ha utilizado el YTONG como muro sustentante.

2." Viviendas para la Obra Sindical del Hogar, en Utiel y Algemesí (Empresa Constructora Asturiana).

Conjunto de viviendas ejecutadas con muros sustentantes de YTONG.

3. Mutua de Viajantes y Representantes, Valencia (Empresa Hidrocivil).

Esta obra es de estructura metálica, utilizándose el YTONG para cierre de fachadas.

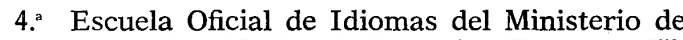
Educación Nacional, Valencia (Empresa Hidrocivil).

5. Escuela de Ingenieros Agrónomos del Ministerio de Educación Nacional, Valencia (Empresa Cleop).

6." Parador Nacional de Turismo de La Dehesa, Valencia (Empresa Jalón, S. L.).

7.a $\quad$ Factoría de Perlofil, Alcalá de Henares (Empresa Entrecanales y Távora).

8. Fábrica de cemento Cía. Valenciana de Cementos Portland, S. A., Buñol (Empresa Sicop).

9. Fábrica Fertiberia, Castellón (Empresa Entrecanales y Távora).

10. Fábrica Fertiberia, Huelva (Empresa Alcisa).

11. Refinería de Petróleos Esso, Castellón (Empresa Agromán).

\section{PRINCIPALES CONCLUSIONES FORMULADAS} POR LA COMISION DE EXPERTOS

7.1. Los bloques YTONG-0,65 de hormigón celular ligero tratados al autoclave, quedan dentro del campo de aplicación que fijan las «reglas para apreciación técnica de bloques para muros de hormigón celular ligero tratados al autoclave».

7.2. Las características físicas y mecánicas de los bloques YTONG-0,65, determinadas mediante ensayo por el Instituto Eduardo Torroja, satisfacen las prescripciones que se fijan en las reglas antes mencionadas, tal y como figura en el Cuadro.

7.3. Por parte del fabricante este material queda sometido al debido control periódico en laboratorio.

7.4. Por tanto, los bloques YTONG-0,65 fabricados por SAMO pueden considerarse técnicamente aptos para la construcción de muros resistentes y de cerramiento, con las siguientes observaciones:

7.4.1. En zonas de acusado rigor higrotérmico es aconsejable, como en otros materiales tradicionales (bloques de mortero, hormigones, ladrillos, etcétera), protegerlos con un revestimiento exterior impermeable (película transparente, pintura, enfoscado, etc.)

7.4.2. Como en el caso de edificios construidos con otros materiales de la misma naturaleza (fábricas de ladrillo, por ejemplo), la conservación de estos revestimientos queda subordinada a la ausencia total de asientos diferenciales del edificio, lo que exige por parte del constructor un cuidado especial en el establecimiento de las cimentaciones, así como en la ejecución de los zunchos de atado.

7.4.3. Desde el punto de vista de la estabilidad, debe señalarse que el Documento de Idoneidad Técnica ampara las características técnicas de los bloques en sí, sin prejuzgar su comportamiento dentro de un conjunto estructural resistente. Por consiguiente, su utilización para la construcción de muros que hayan de soportar cargas de consideración debe hacerse adoptando las medidas necesarias para garantizar la resistencia y estabilidad del conjunto.

7.4.4. Dadas las características físicas y químicas del material YTONG, no deberán utilizarse armaduras embebidas en los propios bloques, aunque éstas queden protegidas por un hormigón normal, excepto cuando se tomen las debidas precauciones para evitar la corrosión de las mismas.

7.4.5. Siempre que las condiciones climatológicas lo aconsejen, el almacenamiento de los bloques debe hacerse al abrigo de la intemperie.

\section{DEGISION NUM. 11}

El Director del Instituto Eduardo Torroja de la Construcción y del Cemento,

— en virtud del Decreto 3.652, de 26 de diciembre de 1963, de la Presidencia del Gobierno, por el que se faculta al Instituto Eduardo Torroja de la Construcción y del Cemento para extender el Documento de Idoneidad Técnica de los materiales y procedimientos no tradicionales de construcción utilizados en la edificación y obras públicas,

- de acuerdo con los Estatutos de la Union Européenne pour l'Agrément Technique dans le Construction (UEAtc), 
- considerando la solicitud formulada por la casa SAMO, con domicilio social en Valencia, calle Colón número 68, para renovar el Documento de Idoneidad Técnica número 2, correspondiente a los bloques para muros, en hormigón celular ligero tratados al autoclave, YTONG-0,65, fabricados por dicha empresa,

- y teniendo en cuenta el informe técnico precedente y los ensayos efectuados por el I.E.T.c.c., así como las observaciones de la correspondiente Comisión de Expertos,

\section{E G I D E :}

1. Renovar el Documento de Idoneidad Técnica número 2 para los bloques de hormigón celular ligero tratados al autoclave YTONG-0,65, fabricados por la casa SAMO, de Valencia, y, por consiguiente, reconocer dichos bloques adecuados para su utilización como «elementos para la construcción de muros resistentes y de cerramiento», con las condiciones siguientes:

\section{a) Fabricación}

La fabricación de los bloques YTONG-0,65 deberá satisfacer lo especificado en las REGLAS PARA APRECIACION TECNICA DE BLOQUES PARA MUROS EN HORMIGON CELULAR LIGERO TRATADOS AL AUTOCLAVE, facilitadas en su día por el Instituto Eduardo Torroja a la casa SAMO, en especial en lo que se refiere a los controles sobre materias primas, fabricación y producto acabado.

\section{b) Características del producto}

- en zonas de acusado rigor higrotérmico es aconsejable que los paramentos exteriores de muros realizados con YTONG, se protejan con un revestimiento impermeable (película transparente, pintura, enfoscado, etc.);

- teniendo en cuenta las características físicas y químicas del material YTONG, no deberán utilizarse armaduras embebidas en los propios bloques; excepto cuando se tomen las debidas precauciones para evitar la posibilidad de corrosión de las mismas;

- cuando las condiciones de clima lo aconsejen, el almacenamiento de los bloques, tanto en fábrica como en obra, deberá hacerse al abrigo de la intemperie.

\section{c) Puesta en obra}

Para la puesta en obra de los bloques deberán seguirse las indicaciones dadas en el informe técnico adjunto a esta decisión.

2. El presente Documento de Idoneidad Técnica número 11 anula y reemplaza al Documento de Idoneidad Técnica número 2 de fecha 29 de abril de 1963. Su plazo de validez será de un año a partir del 1 de octubre de 1966.

3. De no existir modificación alguna por parte del fabricante, en lo que respecta a materias primas, fabricación y características del producto durante el período de validez del presente Documento, los bloques de YTONG-0,65 podrán incluirse entre los materiales de carácter tradicional, debiendo cumplir éstos con las especificaciones del Pliego particular de condiciones de fabricación, recepción y puesta en obra que redactará en su día el I.E.T.c.c. al caducar este Documento.

Madrid, 1 de octubre de 1966.

El Director del Instituto Eduardo Torroja,

J. NADAL 


\begin{tabular}{|c|c|c|c|}
\hline G.B.t.c.c. & \multicolumn{2}{|c|}{$\begin{array}{c}\text { Materiales y procedimientos no tradicionales de construcción } \\
\text { DOCUMENTO DE IDONEIDAD TECNICA }\end{array}$} & C.D.U.: 69.022 .3 \\
\hline \multirow{2}{*}{$\begin{array}{l}\text { institute } \\
\text { Eduarardo tompoja } \\
\text { Costillares-Chamartín } \\
\text { MADRID - } 16 \text { - ESPAÑA }\end{array}$} & \multirow{2}{*}{\multicolumn{2}{|c|}{$\begin{array}{l}\text { Fabricante: } \\
\text { Sociedad Anónima de Materiales y Obras } \mathbf{S} \text { a m } 0 \\
\text { Domicillo Social: Colón, } 68 \\
\text { Fábrica: MISLATA - VALENCIA }\end{array}$}} & D. I. T. N. ${ }^{0} 12$ \\
\hline & & & $\begin{array}{l}\text { Elementos para } \\
\text { muros } \\
\text { Éléments pour murs } \\
\text { Elements for walls }\end{array}$ \\
\hline
\end{tabular}

RECONOCIDO POR LA "UNION EUROPÉENNE POUR L'AGRÉMENT TECHNIQUE DANS LA CONSTRUCTION"

\section{INFORME TECNICO}

\section{MATERIAL}

\subsection{Definición}

E1 YTONG-0,50, es un hormigón ligero del grupo de los hormigones a la cal. Es de estructura porosa, formada por células esferoidales estancas cuyas dimensiones dependen de la densidad. Las paredes de estas células tienen como principal componente el hidrosilicato cálcico formado por tratamiento al vapor.

\section{FABRICACION}

\subsection{Materias primas}

Las materias primas utilizadas son:

- rodeno (roca arenisca con gran porcentaje de sílice),

- cal grasa y

- polvo de aluminio.

Este último en pequeña cantidad.

\subsection{Equipo de fabricación}

El equipo empleado para la fabricación es de origen nacional, a excepción de la máquina cortadora que es de procedencia sueca. Está constituido fundamentalmente por:

En la sección de molido:

- machacadora,

- molinos de martillos y molino de bolas,

- elevadores,

- transportadores helicoidales,

- silos y tolvas.

En la sección de mezclado:

- tolvas,

— básculas y

- una mezcladora.

En la sección de cortado:

- máquina cortadora,

- vagonetas $\mathrm{y}$

- moldes.
En la sección de curado al vapor:

- autoclave y

- generador de vapor.

\subsection{Proceso}

El rodeno y la cal grasa se molturan por separado y posteriormente estos productos son dosificados, mezclados y homogeneizados adicionándoles a continuación agua y polvo de aluminio. La masa flúida así obtenida se vierte sobre moldes metálicos de $2,40 \mathrm{~m}$ de longitud, $1,70 \mathrm{~m}$ de ancho y $0,49 \mathrm{~m}$ de profundidad, en donde las burbujas desprendidas por efecto del polvo de aluminio hacen aumentar el volumen de dicha masa hasta producir un ligero desbordamiento. De esta forma adquiere, después de cierto tiempo en reposo, una consistencia tal que permite cortarlo en bloques. Después de cortados se introducen en un autoclave, donde se someten a la acción del vapor de agua a presión para conseguir las propiedades finales del material.

Por último, los bloques se sacan de los moldes $\mathrm{y}$ son almacenados.

\subsection{Controles}

\subsubsection{Materias primas}

Con independencia del laboratorio general, existe otro laboratorio anejo cuya única misión consiste en el análisis sistemático de las materias primas, en especial del contenido en cal, ya que el rodeno tiene una composición de sílice prácticamente constante.

Análogamente se realiza con el polvo de aluminio, determinando velocidad de reacción.

\subsubsection{Fabricación}

El control de fabricación se extiende al de las primeras materias como se ha indicado, así como al proceso, determinándose finura de los productos molidos, temperatura $\mathrm{y}$ dosificación en el amasado, temperatura de curado al aire y temperatura y presiones en el curado al vapor.

\subsubsection{Producto acabado}

Del producto acabado se determinan con regularidad:

- densidad, 
- resistencia a compresión,

- absorción de agua,

y periódicamente la retracción por desecado.

Para estos controles se obtienen de cada tres moldes de autoclave dos bloques de muestra, uno del centro y otro del borde. De cada bloque se obtienen tres probetas cúbicas de $10 \mathrm{~cm}$ de lado y otras prismáticas. Las primeras se secan hasta un 5 por 100 de humedad relativa/volumen, y se rompen en la prensa para deducir su resistencia a compresión. Las probetas prismáticas se secan a $100^{\circ} \mathrm{C}$ hasta peso constante para determinar la densidad en dicho estado. La media de estos ensayos se reseña en el parte correspondiente.

Para determinar la retracción se utiliza un armario con circulación constante de aire y un grado de humedad adecuado, en el que se colocan las probetas saturadas de agua, midiendo la longitud de las mismas antes de introducirlas en aquél y después de alcanzado el equilibrio con la humedad del aire en circulación.

\section{ELEMENTOS}

Los bloques tienen dimensiones nominales de $49 \times 24 \mathrm{~cm}$, siendo los espesores de $15 ; 18 ; 21$ y 24 centímetros.

Las dimensiones efectivas son $490 \times 238 \mathrm{~mm}$ y los espesores: 148; 178; 208 y 238 milímetros.

\section{PUESTA EN OBRA DEL MATERIAL}

\subsection{Ejecución y acabado de la albañilería}

La puesta en obra del YTONG-0,50 se rige, en general, por las normas tradicionales de la buena albañilería.

Los aparejos en las fábricas con YTONG-0,50 son los normales en construcción, siendo las juntas horizontales y verticales de un centímetro.

Antes de proceder a la puesta en obra de los bloques se aconseja mojarlos. En tiempo normal es suficiente con que el agua penetre unos $5 \mathrm{~mm}$ a partir de la superficie de las juntas, mientras que en tiempo seco el agua debe penetrar hasta 8 ó $10 \mathrm{~mm}$, con el fin de evitar el riesgo de deshidratación del mortero.

Los bloques se pueden cortar con herramienta es. pecial, así como serrar con un serrucho de púas gruesas o trabajar con hacha y formón. Esto, por ejemplo, permite preparar las mochetas necesarias para la colocación de cercos de ventanas y puertas. También, por procedimientos muy simples pueden ejecutarse las rozas para empotrar la instalación eléctrica y hacer los huecos para colocar los cajetines de empalme e interruptores. Otra de las cualidades del material es la de permitir la fijación de tornillos sin necesidad de utilizar tacos especiales.

\subsection{Morteros}

Para la puesta en obra de los bloques se utilizan los morteros que habitualmente se emplean en albañilería. Sin embargo, se recomienda que, dada la marcada rugosidad de las superficies de los bloques, dichos morteros sean de consistencia plástica.

En la ejecución de la tabiquería se puede utilizar la pasta de yeso como elemento de unión.

\subsection{Revestimientos}

Para la ejecución de los revestimientos pueden emplearse los mismos morteros que para la ejecución de las fábricas de bloques.

Es conveniente dejar secar la fábrica antes de la ejecución de los revestimientos, así como frotar las superficies a revestir con un cepillo de púas metálicas o similar, con objeto de quitar las partículas de material que puedan haber quedado sueltas después del cortado en fábrica.

Sin embargo, si el tiempo es muy seco cuando vayan a hacerse los revestimientos, debe mojarse la superficie de YTONG hasta una profundidad de unos 5 milímetros.

\section{ENSAYOS PARA RENOVACION DEL DOCUMEN-} TO DE IDONEIDAD TECNICA

Para proceder a la renovación del D.I.T. número 3, las muestras obtenidas en fábrica por un representante del Instituto Eduardo Torroja fueron sometidas a ensayo de acuerdo con lo especificado en las «Reglas para apreciación técnica de bloques para muros en hormigón celular, tratados al autoclave». El resumen de los resultados obtenidos en esos ensayos y los valores exigidos por las referidas reglas se recogen en el cuadro adjunto.

\begin{tabular}{|c|c|c|c|}
\hline \multicolumn{2}{|r|}{ CA RACTERIS TICAS } & $\begin{array}{l}\text { Resultados } \\
\text { de los ensayos }\end{array}$ & Reglas \\
\hline & Precisión dimensiones & -3 & \pm 3 \\
\hline \multirow{2}{*}{$\begin{array}{l}\text { Resistencia } \\
\text { a compresión }\end{array}$} & Resistencia media, en $\mathrm{kg} / \mathrm{cm}^{2}$ & 37,2 & 35 \\
\hline & Dispersión de resultados (coeficiente de variación, en \%) & 14 & 15 \\
\hline \multirow{2}{*}{ Densidad } & Diferencia entre densidad nominal y en estado seco & $+0,009$ & $\pm 0,05$ \\
\hline & Dispersión de resultados (coeficiente de variación, en \%) & 2,7 & 3 \\
\hline \multicolumn{2}{|c|}{ Variación dimensional higrométrica, en $\mathrm{mm} / \mathrm{m}$} & 0,488 & 0,65 \\
\hline
\end{tabular}




\section{REFERENCIAS DE UTILIZACION}

Construcciones Mir y Ruiz Barón de Cárcer, 48

VALENCIA

Juan Vinyas, Ing.

Paseo de San Juan, 34

BARCELONA

D. Elante Massa

San Vicente, 99

VALENCIA

Cía. Valenciana Cementos

Portland, S. A.

Colón, 68

VALENCIA

D. José Bellver Gil

Alfambra, 11

VALENCIA

Industrias Roser

San Rafael, 10

VALENCIA
Obra: c/ Conchita Piquer

Utilización: Cierre fachadas en edificios con estructura.

Obra: Casás de la Selva

Utilización: Aislamiento de hornos.

Obra: Noviciado Dominicos en Vedat (Torrente)

Utilización: Aislamiento de terrazas.

Obra: Factoría «C» (Buñol)

Utilización: Cierre naves industriales.

Obra: Varias.

Utilización: Aislamiento hornos.

Obra: Varias.

Utilización: Aislamiento hornos.

\section{OBSERVACIONES DE LA COMISION DE EXPERTOS}

1. Los bloques YTONG-0,50 de hormigón celular tratados al autoclave, quedan dentro del campo de aplicación que determinan las «Reglas para la apreciación técnica de bloques para muros, de hormigón celular ligero tratados al autoclave».

2. Las características físicas y mecánicas de los bloques YTONG-0,50, determinadas mediante ensayo sobre las muestras tomadas en fábrica por un representante del I.E.T.c.c., satisfacen las reglas mencionadas anteriormente en todas sus condiciones como puede apreciarse en el cuadro.

3. El fabricante ejerce sobre este material un control periódico en laboratorio.

4. En función de las características físicas del material pueden deducirse las siguientes observaciones y recomendaciones.

4.1. En zonas de acusado rigor higrotérmico es aconsejable, como con cualquier otro material poroso (ladrillos, bloques de mortero, etc.), proteger las fábricas de YTONG con un revestimiento exterior impermeable (película transparente, pintura, enfoscados, etc.).
4.2. La conservación de estos revestimientos queda subordinada, como en el caso de fábricas realizadas con otros materiales, a la ausencia de asientos diferenciales del edificio, lo que obliga al constructor a cuidar la ejecución y establecimiento de las cimentaciones y zunchos de atado.

4.3. Dadas las características físicas y químicas del material YTONG, no deben utilizarse armaduras embebidas en los bloques, aunque éstas queden protegidas por un hormigón normal, excepto cuando se tomen las debidas precauciones para evitar la posibilidad de corrosión de las armaduras.

4.4. Siempre que las condiciones climatológicas lo aconsejen, el almacenamiento de los bloques debe hacerse al abrigo de la intemperie.

4.5. Finalmente, debe señalarse que el presente Documento de Idoneidad Técnica ampara las características técnicas del material en sí, pero no se refiere al comportamiento necesario del mismo dentro de un conjunto estructural resistente. Por tanto, su utilización en muros que hayan de soportar cargas importantes, debe hacerse adoptando las medidas necesarias para garantizar la resistencia y estabilidad del conjunto.

\section{DECISION NUM. 12}

\section{EL DIRECTOR DEL INSTITUTO EDUARDO TORROJA DE LA CONSTRUCCION Y DEL CEMENTO,}

- en virtud del Decreto 3.652, de 26 de diciembre de 1963, de la Presidencia del Gobierno, por el que se faculta al I.E.T.c.c. para extender el Documento de Idoneidad Técnica de los materiales y procedimientos no tradicionales de construcción utilizados en la edificación y obras públicas, 
- de acuerdo con los Estatutos de la Union Européenne pour l'Agrément Technique dans la Construction (U.E.A.t.c.),

- teniendo en cuenta lo especificado en la Decisión número 3 de fecha 1 de mayo de 1963,

- considerando la petición formulada por la Empresa SAMO, con domicilio social en la calle Colón, número 68, de Valencia, para renovación del Documento de Idoneidad Técnica número 3, expedido por el Instituto Eduardo Torroja con fecha 29-5-63, correspondiente a los bloques para muros, en hormigón celular ligero tratados al autoclave, YTONG-0,50.

- y a la vista del informe y ensayos realizados por el Instituto Eduardo Torroja sobre nuevas muestras obtenidas en fábrica, así como las precedentes observaciones de la Comisión de Expertos,

\section{DEGIDE:}

1. Renovar el D.I.T. número 3 correspondiente al material YTONG-0,50, con un plazo de validez de veintisiete meses a partir de $10^{\circ}$ de octubre de 1966.

2. Considerar a los bloques para muros en hormigón celular ligero tratados en autoclave YTONG-0,50, fabricados por la Empresa SAMO en su fábrica de Mislata (Valencia), y definidos por el informe técnico adjunto, aptos para ser empleados como "elementos para la construcción de muros resistentes y de cerramiento" con las siguientes condiciones:

\section{a) Fabricación}

La fabricación de los bloques YTONG-0,50 deberá satisfacer en todo momento lo especificado en las «Reglas para apreciación técnica de bloques para muros en hormigón celular ligero tratados al autoclave», concretamente en lo que se refiere al control de materias primas, y producto acabado, con el fin de mantener la homogeneidad manifestada en los ensayos para el D.I.T., cuyo resumen figura en el informe técnico adjunto.

\section{b) Características del producto}

- en edificios situados en zonas de extremado rigor higrotérmico, construidos con muros de YTONG, es aconsejable proteger los paramentos exteriores con un revestimiento impermeable (película transparente, pintura, enfoscado, etc);

- teniendo en cuenta sus características físicas y químicas no deberán emplearse armaduras embebidas en los propios bloques, si no se toman las debidas precauciones para evitar la posible corrosión de las mismas;

- cuando las condiciones climatológicas así lo aconsejen, el almacenamiento de los bloques, tanto en fábrica como en obra, deberá hacerse al abrigo de la intemperie.

3. La validez del presente documento caduca el 31 de diciembre de 1968.

Madrid, 28 de septiembre de 1966

El Director del Instituto Eduardo Torroja,

J. NADAL 